\title{
Limiar anaeróbio determinado pelo teste do lactato mínimo em ratos: efeito dos estoques de glicogênio muscular e do treinamento físico
}

\author{
Fabrício A. Voltarelli \\ Maria A. R. Mello \\ Claudio A. Gobatto
}

\author{
Universidade Estadual Paulista "Júlio de Mesquita Filho" \\ Campus de Rio Claro, Departamento de Educação Física \\ Instituto de Biociências, Brasil
}

\begin{abstract}
RESUMO
O presente estudo visou verificar, em ratos: a) o efeito do teor de glicogênio muscular sobre o limiar anaeróbio estimado pelo teste do lactato mínimo e b) avaliar a sensibilidade do teste às alterações do condicionamento aeróbio decorrentes do treinamento físico. Para avaliar os efeitos dos estoques de glicogênio muscular, o teste foi realizado nos animais no estado alimentado (controle) e após jejum de 12 horas. Em outra série de experimentos, os ratos foram avaliados em 3 situações: Sedentária (controle) e Treinamento por 4 e 8 semanas. Os ratos treinados nadaram 5 dias por semana, 1 hora por dia, suportando sobrecarga (\% peso corporal - pc) equivalente ao Lan individual. Os valores médios do limiar anaeróbio nos estados Jejum $(n=7)$ e Alimentado $(n=7)$ foram de 4,90 $\pm 0,08$ e $4,88 \pm 0,06 \%$ do pc a $5,51 \pm 0,37$ e $6,65 \pm 0,27 \mathrm{mmol} / 1$ de lactato sanguíneo, respectivamente. Os valores médios do limiar anaeróbio na situação sedentária $(n=16 ; 4,93 \pm 0,07 \%$ do pc a $7,39 \pm 0,39 \mathrm{mmol} / \mathrm{L}$ de lactato sanguíneo) foram melhorados com o treinamento $(n=16 ; 5,98 \pm 0,02 \%$ do pc a $5,89 \pm 0,14$ $\mathrm{mmol} / \mathrm{L}$ de lactato sanguíneo, após 8 semanas). A depleção do glicogênio muscular não alterou a carga de trabalho equivalente ao limiar anaeróbio, mas reduziu a concentração sanguínea de lactato na qual o mesmo apareceu. O limiar determinado pelo teste do lactato mínimo foi sensível às modificações do condicionamento físico aeróbio.
\end{abstract}

Palavras-chave: limiar anaeróbio, estoque de glicogênio muscular, treinamento físico, ratos, natação.

\begin{abstract}
Anaerobic threshold determined by lactate minimum test in rats: Effect of muscle glycogen stores and of physical training

The present study aimed to verify, in rats: a) the effect of the muscle glycogen stores on the anaerobic threshold determined by lactate minimum test and b) to evaluate the sensitivity of the test to the alterations of the aerobic conditioning induced by physical training. For the evaluation of the influence of muscle glycogen stores, the test was performed by the animals in the fed state (control) and after a 12 hour fast. In another set of experiments, the animals were evaluated in 3 situations: Sedentary (control) and after 4 and 8 weeks training. Trained rats swan 5 days per week, 1 hour per day, supporting overload (\% body weight - bw) equivalent to the individual anaerobic threshold. The mean values of the anaerobic threshold after fasting $(n=7)$ and in the fed state $(n=7)$ were of $4.90 \pm 0.08$ and $4.88 \pm 0.06 \%$ of bw at concentrations of $5.51 \pm 0.37$ and $6.65 \pm 0.27 \mathrm{mmol} / \mathrm{l}$ of blood lactate, respectively. The mean values of anaerobic threshold in the sedentary situation $(n=16 ; 4.93 \pm 0.07 \%$ of bw at $7.39 \pm 0.39 \mathrm{mmol} / \mathrm{L}$ of blood lactate) were improved by training $(n=16 ; 5.98 \pm 0.02 \%$ of $b w$ to $5.89 \pm 0,14 \mathrm{mmol} / \mathrm{L}$ of blood lactate, after a 8 week period). The depletion of the muscle glycogen stores did not modify the workload equivalent to the anaerobic threshold, but reduced the blood lactate concentration in which it appeared. The threshold determined by the lactate minimum test was sensible to modifications of the aerobic physical conditioning.
\end{abstract}

Key Words: anaerobic threshold, muscle glycogen stores, physical training, rats, swimming. 


\section{INTRODUÇÃO}

O Limiar anaeróbio (Lan), definido como a carga de trabalho na qual o lactato sanguíneo começa a se acumular desproporcionalmente durante exercícios progressivos, é considerado bom indicador do condicionamento aeróbio e tem sido utilizado na prescrição de treinamento em diferentes modalidades de exercício $(7 ; 9 ; 14 ; 21)$.

Tegtbur et al (18) desenvolveram um protocolo de teste para a determinação do Lan, denominado teste do lactato mínimo (TLM). Esse teste envolve a realização de exercício supramáximo, por um breve período de tempo, visando a indução da hiperlactacidemia antes do início do teste padrão com cargas progressivas em esteira rolante. O Lactato sanguíneo mínimo (LSM) foi definido como a velocidade na qual a curva em forma de "U", obtida com os valores de lactato sanguíneo durante o teste progressivo, atinge o nadir. Esse valor mínimo de lactato sanguíneo supostamente indica o Lan (8).

Por razões óbvias, grande número de pesquisas envolvendo o exercício físico tem sido conduzido em animais de laboratório, especialmente o rato e as concentrações de lactato, utilizadas para a determinação da intensidade de esforço. Como existem diferenças metabólicas entre seres humanos e ratos, é razoável especular sobre potenciais e diferenças entre espécies com respeito ao fluxo de lactato e outras variantes durante o exercício. A despeito da importância do problema, ainda são raros os estudos que tratam da cinética de lactato em ratos durante o exercício.

Uma vez que a determinação do lactato mínimo requer apenas um teste realizado em um único dia, poderia ser adequado à obtenção do Lan em ratos durante exercício de natação. Dessa forma, nosso grupo desenvolveu recentemente estudos visando padronizar um protocolo para a determinação do Lan em ratos durante a natação, utilizando os princípios do TLM estabelecidos por Tegtbur et al (18). Em nosso estudo, o Lan médio calculado dos animais foi obtido na carga de $4,95 \pm 0,10 \%$ do peso corporal à concentração sanguínea de lactato de $7,17 \pm 0,16 \mathrm{mmol} / \mathrm{L}(19)$.

Existem, ainda, pontos que permanecem obscuros e necessitam de esclarecimento antes do emprego, com finalidades práticas, do TLM para estimar o Lan em ratos. Conforme demonstrado em seres humanos, a depleção dos estoques de glicogênio muscular pode reduzir a concentração de lactato sanguíneo a uma dada carga de exercício (18). Com base nessas informações, seriam a intensidade de exercício e a concentração sanguínea de lactato equivalentes ao Lan determinado pelo TLM, em ratos, afetadas pela redução do glicogênio muscular? Além disso, seria o Lan assim determinado sensível às modificações do condicionamento físico aeróbio ocasionadas pelo treinamento físico nesses animais? Dessa forma, o presente estudo foi delineado com a finalidade de: a) verificar se o Lan determinado pelo teste do lactato mínimo sofre influência da redução dos estoques de glicogênio muscular (induzido por jejum de 12 horas); e b) avaliar a sensibilidade do teste do lactato mínimo às alterações do condicionamento aeróbio decorrentes do treinamento em exercício de natação na carga equivalente ao Lan individual determinada em ratos.

\section{MATERIAIS E MÉTODOS \\ Animais}

Foram utilizados ratos da linhagem Wistar, machos, adultos (90-100 dias de idade), eutróficos e sedentários, cujas mães eram provenientes do Biotério Central da Universidade Estadual Paulista (UNESP) - Botucatu/SP. Os animais permaneceram em gaiolas coletivas (5 ratos por gaiola) e foram alimentados com ração comercial (Purina ${ }^{\circledR}$ ) para roedores e água ad libitum bem como mantidos sob ciclo periódico claro e escuro de 12 horas à temperatura média de $25 \pm 2^{\circ} \mathrm{C}$. Todos os experimentos com os animais foram realizados de acordo com a European Convention for Protection of Vertebrate Animals used for Experimental and other Scientific Purposes (Council of Europe ${ }^{\circ} 123$, Strasburg, 1985).

\section{Adaptação ao meio líquido}

A adaptação consistiu em manter o animal em contato com água rasa à temperatura de $32 \pm 2^{\circ} \mathrm{C}$, durante 3 semanas, 5 dias por semana por 30 minutos. Nas $2^{\text {a }}$ e $3^{\text {a }}$ semanas de adaptação, os mesmos suportaram sobrecarga equivalente a $5 \%$ do peso corporal (19). O propósito da adaptação foi reduzir o estresse dos animais frente ao exercício físico realizado na água. 


\section{Teste do Lactato Mínimo (TLM)}

A determinação do Lan pelo TLM, conforme proposto para humanos $(8 ; 18)$, consiste em: a) um período breve de exercício de alta intensidade para provocar um aumento de lactato sanguíneo circulante; b) um período breve de recuperação para assegurar a hiperlactacidemia; e c) um protocolo de teste de exercício com cargas progressivas, com coleta de sangue para análise de lactato a cada carga. Uma vez que a porção progressiva do TLM começa quando o sujeito apresenta níveis altos de lactato sanguíneo, a mesma produz um perfil de lactato sanguíneo característico, em forma de "U". O LSM foi definido como a intensidade de exercício na qual a "curva em forma de U", derivada dos valores de lactato sanguíneo obtidos durante o TLM, atinja o nadir. Partindo dos princípios desse protocolo, o teste foi adaptado em nosso laboratório para as condições do rato (19) e empregado no presente estudo, com pequenas modificações, para a determinação do Lan dos animais.

\section{TLM adaptado às condições do rato}

Inicialmente, os animais foram colocados no tanque cheio de água, suportando uma sobrecarga equivalente a $50 \%$ do peso corporal e exercitaram-se, anaerobiamente (saltos), durante 6 minutos (30 segundos de exercício interrompidos por 30 segundos de repouso), para a elevação da concentração de lactato sanguíneo circulante. Após 9 minutos de repouso, os animais iniciaram exercício de natação com intensidades progressivamente maiores $(4,5 ; 5,0 ; 5,5,6,0 \mathrm{e}$ $6,5 \%$ do peso corporal), com duração de 5 minutos de exercício em cada carga. Antes do início do teste (repouso) e a cada troca de carga, foram coletadas amostras de sangue $(25 \mu \mathrm{l})$, através de pequeno corte na extremidade distal da cauda, para a determinação do lactato no Analisador Eletroquímico YSL $\AA$ modelo 1500 SPORT, Yellow Spring, OH, USA (19). Ao serem retirados da água para as coletas de sangue, corpo e cauda dos animais foram cuidadosamente secos com uma toalha, para evitar contaminação e diluição das amostras. A curva lactato sanguíneo vs carga de trabalho, para cada rato, determinada no teste, foi obtida por ajuste polinomial de grau 2 , com auxílio de um programa de computador (MICROSOFT OFFICE - EXCEL $®$ ). Procedeu-se, então, à determinação matemática, pela análise da função, do menor valor da concentração de lactato sanguíneo e respectiva carga de trabalho. Esse menor valor indicou o Lan individual.

\section{Jejum}

Tegtbur et al (18) demonstraram em sujeitos humanos que embora os valores de lactato sanguíneo sejam mais baixos em condições de depleção do glicogênio muscular isso não afeta a intensidade equivalente ao Lan determinado pelo TLM. Essa série de testes foi efetuada para verificar se estoques normais ou baixos de glicogênio muscular nos ratos antes do início da aplicação do teste, alterariam a intensidade de exercício equivalente ao Lan. Para isso, os animais foram submetidos a jejum de 12 horas, condição que sabidamente depleta os estoques de glicogênio muscular e avaliados quanto ao Lan através do TLM. Imediatamente após a realização do teste, os animais foram sacrificados e o músculo sóleo da pata direita foi removido para determinação das concentrações de glicogênio (15). Animais no estado alimentado (grupo controle) foram simultaneamente submetidos ao TLM, sacrificados e analisados quanto ao teor de glicogênio do músculo sóleo. Para fins de comparação, foi também avaliado o teor de glicogênio no músculo sóleo de ratos no estado alimentado e/ou após jejum de 12 horas, sacrificados em repouso.

\section{Treinamento físico}

Esse procedimento foi realizado utilizando-se outro lote de animais e teve como propósito verificar a sensibilidade do teste do lactato mínimo às mudanças do condicionamento aeróbio decorrentes do treinamento. Para isso, os ratos foram submetidos por 8 semanas a exercícios de natação em tanques coletivos $(100 \mathrm{~cm} \times 80 \mathrm{~cm} \times 80 \mathrm{~cm})$, contendo água a $30-32^{\circ} \mathrm{C}, 5$ dias por semana, suportando sobrecarga equivalente ao Lan individual. Ao final do período de 4 semanas de treinamento os animais foram novamente submetidos ao TLM. Esse procedimento foi realizado afim de se ajustar a carga de treinamento equivalente ao Lan individual dos ratos (necessário para dar continuidade ao processo de treinamento) e verificar possíveis modificações na cinética do lactato sanguíneo decorrentes do treinamento físico. Finalizadas as 8 semanas de treinamento, os animais foram mais uma vez avaliados pelo TLM, para identificação das mudanças no condicionamento aeróbio (carga de exercício/\% do peso corporal e concentração de lactato sanguíneo/mmol/l). 
Dessa forma, para avaliar os efeitos do treinamento físico, os animais foram avaliados em 3 situações: Sedentária (controle), Treinamento por 4 semanas e Treinamento por 8 semanas. Os mesmos animais foram submetidos a todas as situações.

Teste de natação com carga fixa equivalente ao Lan Foi realizado um teste de natação onde os animais suportaram uma carga fixa equivalente ao Lan durante 30 minutos (4), visando verificar se o lactato sanguíneo estabilizar-se-ia nessa intensidade de exercício pois, teoricamente, o Lan coincide com a máxima fase estável de lactato (1). Foram coletadas amostras sanguíneas durante o teste para a determinação do lactato a cada 5 minutos de exercício. Esse procedimento experimental foi efetuado 48 horas após os animais realizarem o primeiro (condição sedentária), o segundo (4 semanas de treinamento) e o terceiro (final da etapa de treinamento de 8 semanas) TLM.

\section{Análise estatística}

Os dados foram expressos como média \pm desvio padrão. Os procedimentos estatísticos incluíram Análise de Variância (ANOVA), seguida de BONFERRONI, onde apropriado. O nível de significância foi de $\mathrm{p}<0,05$.

\section{RESULTADOS}

\section{Jejum}

A Figura 1 mostra os valores do Lan calculados através da equação polinomial de grau 2 que definiu a curva lactato sanguíneo (LS) (mmol/l) vs carga de trabalho (\% peso corporal-pc), para um rato em cada situação durante o TLM de natação. O Lan individual calculado do animal no estado Alimentado (controle) foi obtido na carga de $4,97 \%$ do pc, na concentração de $6,61 \mathrm{mmol} / 1$ de LS. Em relação ao animal em Jejum, o Lan individual calculado ocorreu na carga de $4,99 \%$ do pc à concentração de $5,94 \mathrm{mmol} / 1$ de LS. Os valores médios do Lan calculados para as situações Jejum $(n=7)$ e estado controle Alimentado $(n=7)$ foram de 4,90 $\pm 0,08$ e $4,88 \pm 0,06 \%$ de carga em relação ao peso corporal, nas concentrações de 5,51 $\pm 0,37$ e 6,65 $\pm 0,27$ $\mathrm{mmol} / \mathrm{L}$ de lactato sanguíneo, respectivamente.
Estado Alimentado [controle]

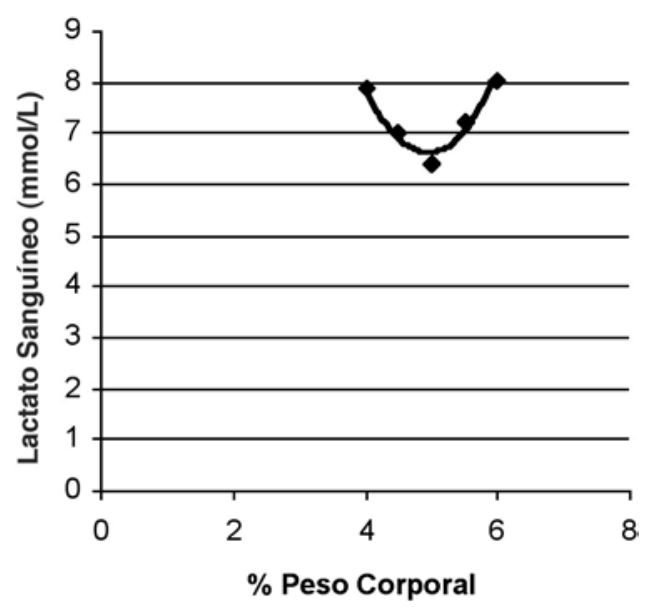

Jejum

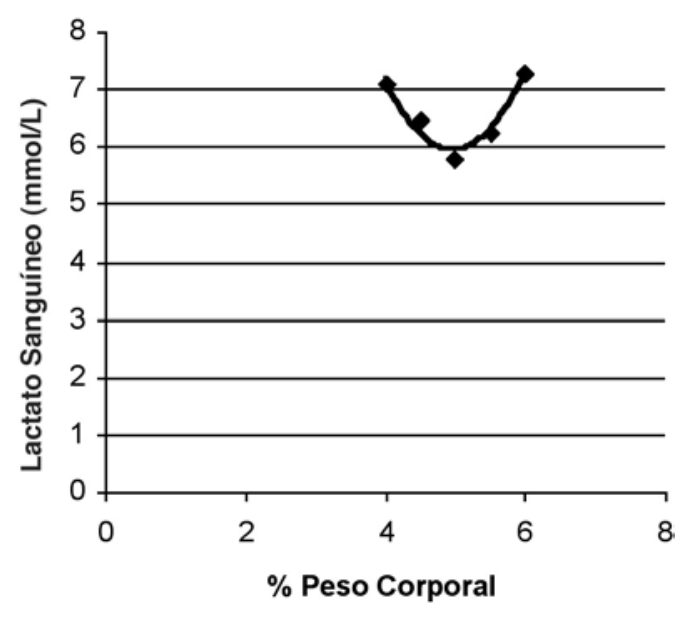

Figura 1: Curva lactato sanguíneo vs carga de trabalho durante teste do lactato mínimo de um rato no estado Alimentado (controle] e de um rato em Jejum. As curvas foram obtidas por ajuste polinomial de grau 2, com auxílio

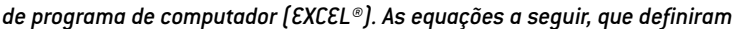
as curvas, possibilitaram os cálculos dos menores valores das concentrações de lactato sanguíneo (LS) e respectivas cargas de trabalho (peso corporal - pc). Para estado Alimentado, $y=1,3886 x 2-13,794 x+40,866$ (6,61 mmol/L de LS à 4,97\% do pc); para Jejum, $y=1,27434 x 2-12,711 x+$ $36,642(5,94 \mathrm{mmol} / \mathrm{L}$ de $L S$ à $4,99 \%$ do $p c)$. Esses menores valores de lactato sanguíneo indicaram as cargas de trabalho equivalentes ao Lan. 
A Tabela 1 mostra as concentrações de glicogênio no músculo sóleo dos animais nas seguintes situações: estado Alimentado (controle), sacrificados após o TLM; estado Alimentado (controle), sacrificados em repouso; Jejum de 12 horas, sacrificados após o TLM e Jejum de 12 horas, sacrificados em repouso. As concentrações médias de glicogênio foram sempre significativamente inferiores nos animais jejuados.

Tabela 1: Teores de glicogênio ( $\mathrm{mg} / 100 \mathrm{mg}$ ] no músculo sóleo dos animais nas situações: estado Alimentado[ $\left.{ }^{\circledR}\right]$ sacrificados em repouso $[A / R$ ); estado Alimentado sacrificados após o TLM (A/TLM); jejum de 12 horas sacrificados em repouso [ $\mathrm{J} / \mathrm{R}$ ] e jejum de 12 horas sacrificados após TLM [J/TLM].

\begin{tabular}{l|c|c|c|c} 
& $\mathrm{A} / \mathrm{R}$ & $\mathrm{A} / \mathrm{TLM}$ & $\mathrm{J} / \mathrm{R}$ & $\mathrm{J} / \mathrm{TLM}$ \\
\hline Média & 0,135 & 0,087 & $0,065^{*}$ & $0,048^{* *}$ \\
Desvio Padrão & 0,035 & 0,005 & 0,003 & 0,003 \\
$\mathrm{n}$ & 5 & $?$ & 5 & $?$
\end{tabular}

$T L M=$ Teste do Lactato Mínimo

${ }^{*} p<0,05$ em relação à situação $A / R$

** $p<0,05$ em relação à situação A/TLM

[ ${ }^{\circledR}$ grupo controle

\section{Treinamento físico}

A Figura 2 mostra os efeitos do treinamento físico de natação sobre o condicionamento aeróbio de apenas um rato suportando carga equivalente ao Lan individual, a título de exemplo. O Lan foi calculado através da equação polinomial de grau 2 que definiu a curva LS (mmol/l) vs carga de trabalho (\% do pc). O Lan individual desse animal, quando em situação Sedentária (controle), foi obtido na carga de 5,05\% do pc a $6,47 \mathrm{mmol} / \mathrm{l}$ de LS. Após 4 semanas de treinamento físico houve melhora nas magnitudes referentes ao Lan individual desse rato, ou seja, o valor da carga de exercício aumentou e a concentração de LS apresentou declínio $(5,41 \%$ do pc a $6,32 \mathrm{mmol} / 1$ de LS). O mesmo comportamento foi observado ao final das 8 semanas de treinamento físico de natação onde o Lan individual foi obtido na carga de $5,97 \%$ do pc na concentração de $5,80 \mathrm{mmol} / \mathrm{l}$ de LS. Os valores médios do Lan calculados para todos os ratos na situação controle Sedentária $(\mathrm{n}=16)$,
Treinamento 4 semanas $(n=16)$ e Treinamento 8 semanas $(n=16)$ foram de 4,93 $\pm 0,07,5,42 \pm 0,01$ e $5,98 \pm 0,02 \%$ de carga em relação ao peso corporal, nas concentrações de 7,39 $\pm 0,39,6,38 \pm 0,12 \mathrm{e}$ $5,89 \pm 0,14 \mathrm{mmol} / \mathrm{L}$ de lactato sanguíneo, respectivamente.

Situação Sedentária (controle)

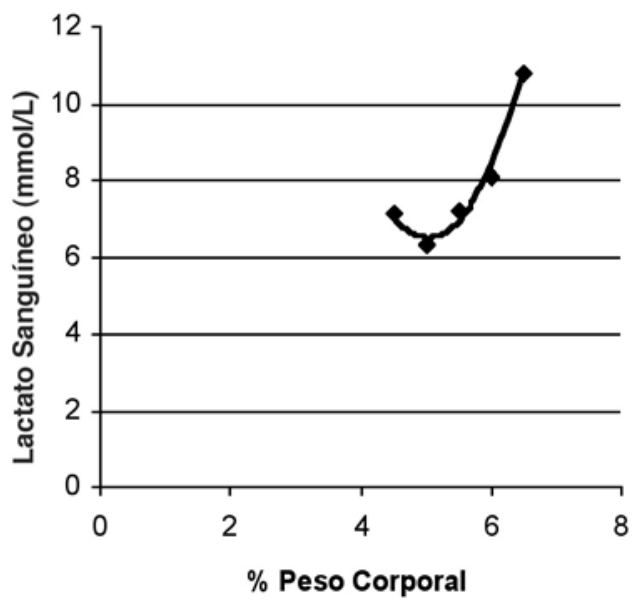

Treinamento 4 Semanas

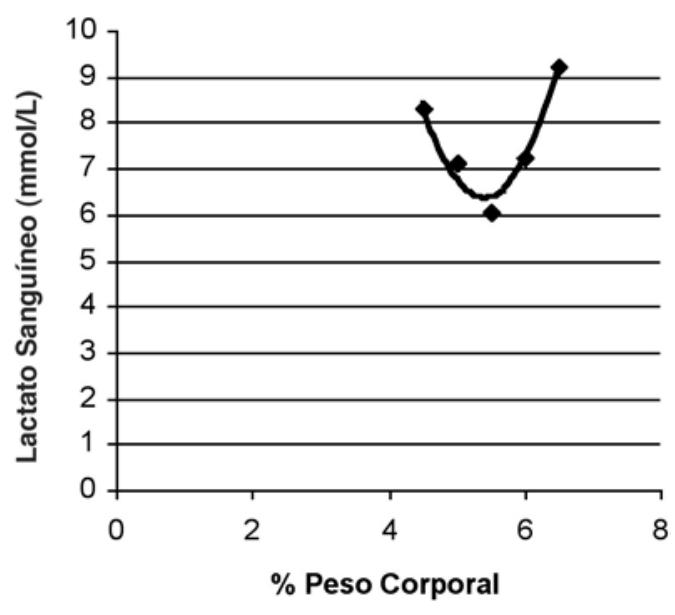


Treinamento 8 Semanas

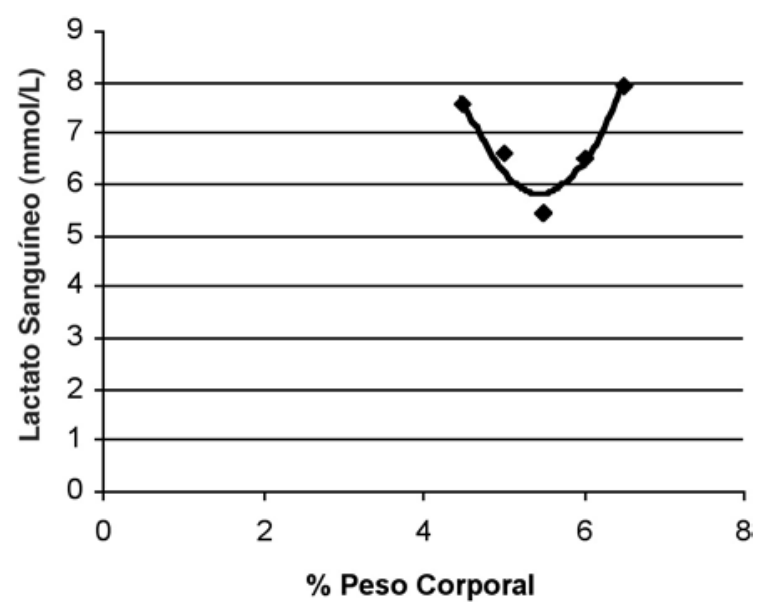

Figura 2: Curvas de lactato sanguíneo vs carga de trabalho de um rato durante testes do lactato mínimo realizados antes (situação controle Sedentária) e após 4 ou 8 semanas de treinamento físico de natação suportando carga equivalente ao Limiar Anaeróbio (Lan] individual. As curvas foram obtidas por ajuste polinomial de grau 2, com auxílio de programa de computador ( $\mathcal{E X C E L ^ { \boxplus }}{ }^{\boxplus}$. As equações a seguir, que definiram as curvas, possibilitaram o cálculo dos menores valores das concentrações de lactato sanguíneo (LS) e respectivas cargas de trabalho (peso corporal - pc). Para situação controle Sedentária, $y=2,0143 x 2-20,339 x+57,81[5,05 \%$ do $p c$ a $6,47 \mathrm{mmol} / \mathrm{L}$ de $L S$ ); para Treinamento 4 semanas $y=2,4343 \times 2-26,405+$ 77,958 ( $5,41 \%$ do pc a $6,32 \mathrm{mmol} / \mathrm{L}$ de $L S$ ) e para Treinamento 8 semanas, $y=2,0229 \times 2-22,119+66,266$ (5,97\% do pc a $5,80 \mathrm{mmol} / \mathrm{L}$ de $L S$ ). Esses menores valores de lactato sanguíneo indicam as cargas de trabalho equivalentes ao Lan em cada situação.

Visando verificar a estabilização do lactato sanguíneo durante o exercício, efetuou-se um teste de natação (30 minutos) com carga fixa equivalente ao Lan individual nas situações Sedentária e após Treinamento por 4 e 8 semanas. Os resultados obtidos para o mesmo animal da Figura 2 são apresentados na Figura 3. Na situação Sedentária, a concentração de LS estabilizou-se em 6,25 $\pm 0,03 \mathrm{mmol} / 1$ na carga de 5,05\% do pc. Após 4 semanas de Treinamento, a concentração de LS estabilizou-se em valor inferior $(6,05 \pm 0,03 \mathrm{mmol} / \mathrm{L})$ quando comparada com a situação controle Sedentária, enquanto que a carga de exercício se elevou $(5,41 \%$ do pc). Após 8 semanas de Treinamento, a concentração de LS esta-

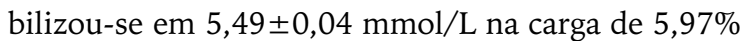
do pc. Fato semelhante foi verificado em relação aos valores médios calculados para o conjunto de animais ( $n=16$ em cada situação), tanto para a carga de trabalho (\% do pc) (situação controle Sedentária= $4,93 \pm 0,09$, Treinamento 4 semanas $=5,42 \pm 0,01 \mathrm{e}$ Treinamento 8 semanas $=5,98 \pm 0,02$ ) quanto para as concentrações médias de lactato sanguíneo (mmol/L) (situação controle Sedentária= $6,66 \pm 0,37$, Treinamento 4 semanas $=6,10 \pm 0,05 \mathrm{e}$ Treinamento 8 semanas $=5,51 \pm 0,15 \mathrm{mmol} / \mathrm{L})$.

Situação Sedentária [controle]

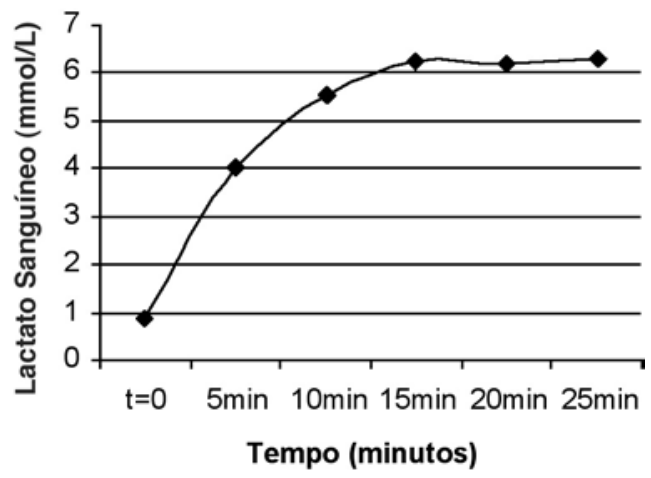

Treinamento 4 Semanas

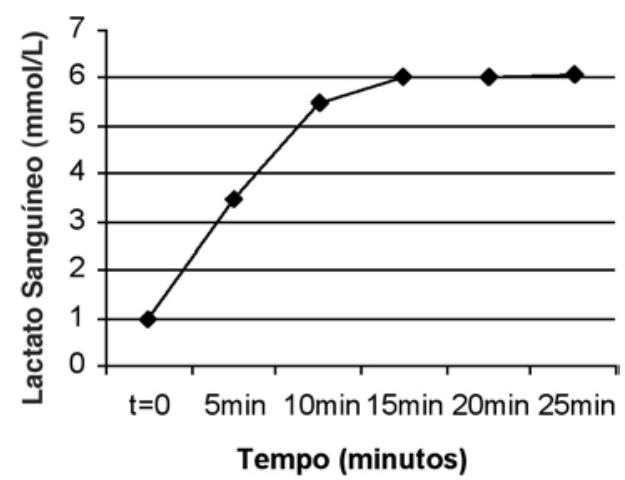


Treinamento 8 Semanas

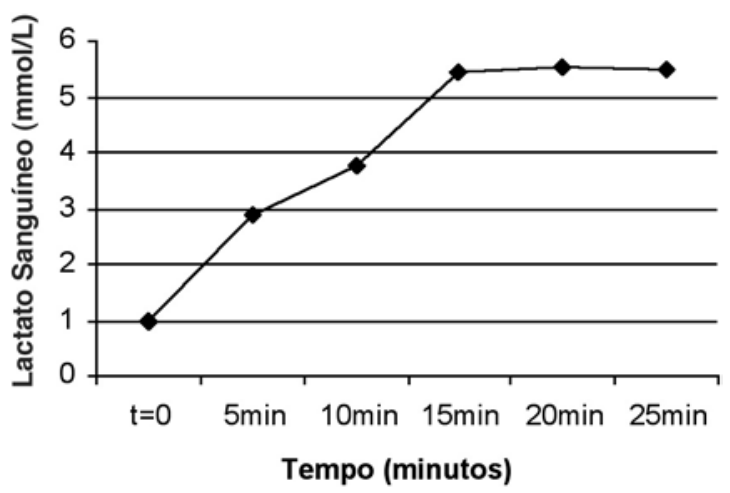

Figura 3: Curva lactato sanguíneo (LS) vs tempo de exercício durante teste de carga fixa de apenas um rato (o mesmo da Figura 2) suportando sobrecarga, em relação ao peso corporal ( $p c$ ), equivalente ao Limiar Anaeróbio [Lan] individual determinado pelo teste do lactato mínimo, nas situações controle Sedentária ( Lan $=5,05 \%$ do pc), Treinamento de 4 semanas (Lan= $5,41 \%$ do pc) e Treinamento de 8 semanas ( $L a n=5,97 \%$ do pc). As concentrações de $L S$ do animal estabilizaram-se em 6,25 $\pm 0,03 \mathrm{mmol} / \mathrm{l}$ quando em Situação Sedentária; 6,05 0,03 $\mathrm{mmol} / \mathrm{l}$ após 4 semanas de treinamento e em $5,49 \pm 0,04 \mathrm{mmol} / \mathrm{l}$ após 8 semanas de treinamento.

\section{DISCUSSÃO}

Apesar das inúmeras tentativas, as bases fisiológicas para o acúmulo de lactato sanguíneo durante o exercício ainda não foram totalmente elucidadas. A despeito disso, a determinação de "limiares" de lactato, isto é, a determinação da intensidade de exercício na qual o lactato sanguíneo começa a se acumular desproporcionalmente, tem-se mostrado ferramenta útil na prescrição de exercícios $(1 ; 7 ; 16)$.

Para fins práticos, o limiar anaeróbio (Lan) tem sido empregado submetendo-se o sujeito a esforços com cargas progressivamente mais elevadas e concomitante avaliação da concentração do lactato sanguíneo. A determinação do Lan pode ser feita baseando-se no desvio da linha de base da concentração circulante de lactato ou na intensidade de trabalho correspondente a uma concentração fixa de lactato circulante. O primeiro método fundamenta-se no fato de que o aumento não linear da concentração de lactato sanguíneo em relação à intensidade do exercício indica a transição do metabolismo, conforme definiram Kinderman et al. (9) e Wasserman \& Mc Ilroy
(21). O segundo procedimento assume o princípio de que, até uma determinada concentração circulante de lactato, ocorre um equilíbrio entre a produção muscular e a remoção desse substrato da circulação, conforme postularam Heck et al. (6).

A determinação do Lan tem-se mostrado também valiosa em estudos clínicos $(13,21)$. Uma vez que existem limitações óbvias nas pesquisas com seres humanos, em especial nos estudos clínicos, os modelos animais têm fornecido importantes informações quanto à realização de exercício físico sob diversas condições experimentais, incluindo obesidade (20), diabetes (11), desnutrição (3) e jejum (17). O presente estudo foi delineado a partir de duas propostas distintas: a) verificar se o Lan determinado pelo teste do lactato mínimo sofre influência da redução dos estoques de glicogênio muscular (induzido por jejum de 12 horas), visto que a depleção do glicogênio muscular pode reduzir a concentração de lactato sanguíneo em uma dada carga de exercício; e b) avaliar a sensibilidade do teste do lactato mínimo às alterações do condicionamento aeróbio decorrentes do treinamento em exercício de natação na carga equivalente ao Lan individual determinada em ratos. No TLM originalmente descrito para seres humanos (18), a acidose láctica inicial foi induzida em atletas por duas corridas exaustivas consecutivas. A segunda corrida foi seguida por 8 minutos de recuperação (andar lento). Em seguida, foi iniciado o teste progressivo de corrida.

Baseando-nos nessas informações, desenvolvemos, em nosso laboratório, um teste para determinar o Lan durante exercício de natação, utilizando os princípios básicos do TLM proposto para seres humanos (18), adaptado às condições do rato (19). O Lan de ratos, nesse último estudo, foi obtido na carga média calculada de $4,95 \pm 010 \%$ do peso corporal (pc) dos animais na concentração média de lactato sanguíneo (LS) calculada de 7,17 $\pm 0,16 \mathrm{mmol} / 1$.

A Figura 1 mostra os valores calculados de intensidade de exercício ( $4,97 \%$ do pc) e lactato sanguíneo $(6,61 \mathrm{mmol} / \mathrm{l})$, correspondentes ao Lan, de apenas um rato no estado Alimentado (controle) do presente estudo. Como se pode notar, a carga de exercício e concentração de lactato sanguíneo, equivalentes ao Lan, obtidos nesse animal, mostraram-se semelhantes aos valores obtidos no estudo anteriormente efetuado com ratos. 
Tegtbur et al. (18) mostram que a depleção dos estoques de glicogênio muscular pode reduzir a concentração de lactato sanguíneo a uma dada carga de exercício durante a realização do TLM por humanos. Os sujeitos deste estudo (18) realizaram o teste com estoques normais (dieta rica em carboidratos + repouso um dia antes do teste, para assegurar que os estoques de glicogênio muscular não fossem depletados) e reduzidos (dieta pobre em carboidrato + exercício de alta intensidade um dia antes do teste, procedimento no qual a redução dos estoques de glicogênio muscular é esperada -2 ;5) de glicogênio muscular. Com base nessas informações, submetemos os animais ao jejum de 12 horas, condição que sabidamente depleta os estoques de glicogênio muscular (10) e os avaliámos quanto ao Lan através do TLM. Observa-se na Tabela 1 que o jejum de 12 horas mostrou-se eficaz em reduzir, significativamente, os estoques musculares de glicogênio $(0,065 \pm 0,003$ $\mathrm{mg} / 100 \mathrm{mg}$ ) quando comparados ao estado controle Alimentado $(0,135 \pm 0,035 \mathrm{mg} / 100 \mathrm{mg})$. Portanto, tal procedimento foi eficiente em mimetizar nos animais a condição de redução de estoque de glicogênio muscular proposta no estudo com seres humanos (18). No estudo realizado por Tegtbur et al (18), a carga de trabalho (velocidade de corrida) correspondente ao Lan dos sujeitos de ambos os grupos (dieta rica em carboidratos $=4,7 \mathrm{~m} / \mathrm{s}$ e pobre em $\mathrm{CHO}=$ $4,7 \mathrm{~m} / \mathrm{s}$ ) não foi modificada quando da realização do TLM. No entanto, a concentração de lactato sanguíneo equivalente ao Lan dos sujeitos foi obtida em níveis consideravelmente inferiores no grupo que recebeu dieta pobre em carboidratos

$(3,1 \pm 1,7 \mathrm{mmol} / \mathrm{l})$ quando comparada ao grupo que recebeu dieta rica nesse substrato $(3,7 \pm 2,2 \mathrm{mmol} / \mathrm{l})$, corroborando achados anteriores da literatura (12). No presente estudo com animais, podemos observar (Figura 1) que o comportamento dos resultados, tanto de carga de trabalho quanto de lactato sanguíneo, foi semelhante ao constatado em seres humanos (18). A carga de trabalho correspondente ao Lan do animal em Jejum (4,99\% do pc) foi semelhante à determinada no animal no estado controle Alimentado ( $4,97 \%$ do pc). No entanto, a concentração de lactato sanguíneo, correspondente ao Lan do animal jejuado $(5,94 \mathrm{mmol} / \mathrm{l})$ apresentou-se significantemente menor em relação ao valor obtido no animal no estado controle Alimentado

$(6,61 \mathrm{mmol} / \mathrm{l})$.

O jejum, sabidamente, reduz a oxidação de carboidratos, a síntese de glicogênio, e, principalmente, a produção de lactato pelo músculo esquelético (10). A Tabela 1 contém resultados que suportam a influência dos estoques de glicogênio muscular na concentração de lactato sanguíneo durante o exercício. Podemos observar que os valores médios dos teores de glicogênio obtidos do músculo sóleo dos animais jejuados submetidos ao TLM ( $\mathrm{n}=7$;

$0,048 \pm 0,003 \mathrm{mg} / 100 \mathrm{mg}$ ) apresentaram-se significativamente menores quando comparados aos valores médios da mesma variável do grupo Alimentado submetido ao TLM ( $\mathrm{n}=7 ; 0,087 \pm 0,005 \mathrm{mg} / 100 \mathrm{mg}$ ). A menor concentração de lactato sanguíneo durante o teste, apresentada pelos animais jejuados, pode ser conseqüência da diminuição no conteúdo total de glicogênio do músculo sóleo em relação aos ratos alimentados. Por outro lado, a intensidade (carga de exercício) equivalente ao Lan não foi afetada pela redução dos estoques musculares de glicogênio. Em outra etapa do presente estudo, o TLM proposto para ratos, foi avaliado quanto à sensibilidade ao treinamento físico aeróbio de natação. Inicialmente, os animais foram submetidos ao TLM para identificação da carga de trabalho (\% do pc) e concentração de LS ( $\mathrm{mmol} / \mathrm{L})$ equivalentes ao Lan dos mesmos. Para ajuste da intensidade de exercício e verificação dos efeitos do treinamento físico, o TLM foi novamente efetuado após 4 e 8 semanas de treinamento físico de natação. Esta situação foi considerada controle para as demais.

Como podemos observar na Figura 2, os valores de LS $(6,47 \mathrm{mmol} / \mathrm{L})$ e carga de exercício $(5,05 \%$ do pc), referentes ao Lan individual do animal na situação controle Sedentária, após o TLM, mostraram-se semelhantes aos obtidos na etapa anterior deste estudo (Figura 1) bem como aqueles obtidos por Voltarelli et al. (19), evidenciando a reprodutibilidade do teste.

Após 4 semanas de treinamento físico (Figura 2), observou-se melhora nos valores referentes ao Lan individual do animal $(5,41 \%$ do pc a $6,32 \mathrm{mmol} / \mathrm{L}$ de LS) quando comparados com os valores na situação controle Sedentária e com os valores encontrados em ratos sedentários por Voltarelli et al. (19). 
Isso é evidência de evolução no condicionamento aeróbio em função do treinamento físico.

A Figura 2 confirma, também, a sensibilidade do TLM, adaptado às condições do rato, ao treinamento físico de natação. Ao final de 8 semanas de treinamento, os valores referentes ao Lan individual do rato $(5,97 \%$ do pc a $5,80 \mathrm{mmol} / \mathrm{L}$ de $\mathrm{LS})$ apresentaram melhora significativa quando comparados com aqueles observados na situação Controle Sedentária, após 4 semanas de treinamento e ainda com os valores obtidos por Voltarelli et al. (19).

Portanto, podemos afirmar que no decorrer das etapas de treinamento físico de natação às quais o animal foi submetido, a carga de exercício equivalente ao Lan elevou-se e a concentração de LS diminuiu. Isto é indicativo de melhoria no condicionamento aeróbio. Essa mesma tendência foi obtida nos valores médios calculados para os animais nas situações controle Sedentária $(\mathrm{n}=16 ; 4,93 \pm 0,07 \%$ do $\mathrm{pc}$ a $7,39 \pm 0,39 \mathrm{mmol} / \mathrm{L}$ de LS), Treinamento por 4 semanas $(\mathrm{n}=16 ; 5,42 \pm 0,01 \%$ do pc a $6,38 \pm 0,12 \mathrm{mmol} / \mathrm{L}$ de LS) e Treinamento por 8 semanas $(n=16 ; 5,98 \%$ do pc a $5,89 \pm 0,14 \mathrm{mmol} / \mathrm{L}$ de LS).

A Figura 3 contém os valores das concentrações de LS de um rato (o mesmo da Figura 2) durante a realização de testes de natação (30 minutos) suportando carga fixa equivalente ao Lan individual do mesmo nas situações controle Sedentária e após $4 \mathrm{e}$ 8 semanas de Treinamento. Estes testes foram efetuados com o intuito de checar a estabilização do lactato sanguíneo durante o exercício realizado na intensidade do Lan, baseando-se no princípio de equilíbrio entre a produção e a remoção do lactato sanguíneo nessa intensidade de esforço (4). Voltarelli et al. (19) realizaram o mesmo teste de carga fixa em ratos eutróficos e sedentários, os quais suportaram sobrecarga equivalente ao Lan determinado pelo TLM e obtiveram estabilização da concentração de LS em 6,41 $\pm 0,30 \mathrm{mmol} / \mathrm{L}$. Valores semelhantes de carga de exercício e estabilização da concentração de LS $(5,05 \%$ do pc a $6,25 \mathrm{mmol} / \mathrm{L})$ foram observados no animal na situação controle Sedentária do presente estudo (Figura 3). Isso sugere que as taxas de produção e remoção do lactato sanguíneo equivaleram-se durante o esforço realizado na intensidade equivalente ao Lan determinado pelo TLM.
Os valores nos quais a concentração de LS estabilizou-se no animal analisado na Figura 3 após 4 e 8 semanas de Treinamento $(6,05 \pm 0,03 \mathrm{mmo} / \mathrm{L} \mathrm{e}$ $5,49 \pm 0,04 \mathrm{mmol} / \mathrm{L}$, respectivamente) foram inferiores à situação controle Sedentária, atestando melhoria no condicionamento aeróbio com o treinamento. Em resumo, a partir dos resultados obtidos, podemos concluir que:

1. O Lan de ratos submetidos à redução dos estoques de glicogênio muscular foi obtido em carga de exercício (\% do peso corporal) semelhante à de ratos controle alimentados. Em contrapartida, a concentração de lactato sanguíneo $(\mathrm{mmol} / \mathrm{l}) \mathrm{na}$ carga de exercício equivalente ao Lan foi menor;

2. O padrão de resposta observada nos ratos foi semelhante aquele descrito na literatura para seres humanos, ou seja, a depleção dos estoques de glicogênio muscular não alterou a carga de trabalho equivalente ao Lan, mas reduziu a concentração de lactato sanguíneo, na qual o mesmo apareceu;

3. O Lan determinado pelo teste do lactato mínimo foi sensível às modificações do condicionamento físico aeróbio dos animais induzidas pelo treinamento;

4. Tomados em conjunto, os resultados do presente estudo indicam a viabilidade da utilização do teste do lactato mínimo na determinação do limiar anaeróbio de ratos.

\section{Agradecimentos}

Os autores agradecem a Clarice Y. Sibuya, José Roberto R. da Silva e Eduardo Custódio pelo excelente suporte técnico e pela indispensável colaboração na coleta de dados. Agradecemos também a Fundação de Amparo à Pesquisa do Estado de São Paulo (FAPESP) pelo apoio financeiro (processo número 02/10296-0).

\section{CORRESPONDÊNCIA}

\section{Fabrício Azevedo Voltarelli}

Departamento de Educação Física, IB, UNESP

Avenida 24-A, 1515

13506-900 Rio Claro, SP, BRASIL

faunesp8@yahoo.com.br 


\section{REFERÊNCIAS}

1. Billat VL, Sirvent P, Koralsztein JP, Mercier J (2003). The concept of maximal lactate steady state: a bridge between biochemistry, physiology and sport science (review). Sports Med 33(6): 407-426.

2. Bonem A, Ness GW, Belcastro AN, Kirby RL (1985). Mild exercise impedes glicogen repletion in muscle. J Appl Physiol 58: 1622-1629.

3. Galdino RS, Souza CCA, Luciano E, Mello MAR (2000). Protein calorie malnutrition does not impair glucose metabolism adaptations to physical exercise. Nutr Res 20: 257-535

4. Gobatto CA, Mello MAR, Sibuya CY, Azevedo JRM, Santos LA, Kokubun E (2001). Maximal lactate steady state in rats submitted to swimming exercise. Comp Biochem Physiol 130 (1): 21-27.

5. Greenhaff PL, Gleeson M, Maughan RJ (1988). Diet-induced metabolic acidosis and the performance of high intensity exercise in man. Eur J Appl Physiol 57: 583-590.

6. Heck HA, Mader G, Hess S, Muck A, Hollman W (1985). Justification of the $4,0 \mathrm{mmol} / \mathrm{L}$ lactate threshold. Int $J$ Sports Med 6: 117-130.

7. Jones AM, Carter H (2000). The effect of endurance training on parameters of aerobic fitness. Sports Med 29: 373-376.

8. Jones AM, Doust JH (1998). The validity of the lactate minimum test for determination of the maximal lactate steady state and physiological correlates to $8 \mathrm{Km}$ running performance. Med Sci Sports Exerc 30: 1304-1313.

9. Kinderman W, Simon G, Keul J (1979). The significance of the aerobic-anaerobic transition for the determination of work load intensities during endurance training. Eur J Appl Physiol 42: 25-34.

10. Li J, Stillman JS, Clore JN, Blackard WG (1993). Skeletal muscle lipids and glycogen mask substrate competition (Randle cycle). Metabolis 42: 451-456.

11. Luciano E, Mello MAR (1999). Atividade física e metabolismo de proteína em músculos de ratos diabéticos. Rev Paul Educ Fís 12: 202-209.

12. Maassen N, Busse MW (1989). The relationship between lactic acid and work load - a measure for endurance capacity or an indicator of carbohydrate deficiency? Eur J Appl Physiol 58: 728-737.

13. Neiva CM, Bunc V, Mello MAR (1999). Glicemia, insulinemia e trigliceridemia de ratos diabéticos experimentais e alimentados com dieta hiperlipídica, submetidos a treinamento físico por aeróbio e anaeróbio. Rev Cient Franca 11: 201-210.

14. Ribeiro L, Balikian P, Malachias P, Baldissera V (2003). Stage length, spline function and lactate minimum swimming speed. J Sports Med Phys Fit 43 (3): 312-318.

15. Siu Lo JCR, Taylor AW (1970). Determination of glycogen in small tissue samples. J Appl Physiol 8(2): 123-132.

16. Sjodin B, Jacobs I (1981). On set of blood lactate accumulation and marathon running performance. Int J Sports Med 2: $23-26$.

17. Stevanato E, Gobatto CA, Sibuya CY, Mello MAR, Kokubun E (1998). Free fatty acid concentration did not affect carbohydrate metabolism in soleus muscle in vitro. Med Sci Sports Exerc 30(5): S247-S247.

18. Tegtbur U, Busse MW, Braumann KM (1993). Estimation of individual equilibrium between production and catabolism during exercise. Med Sci Sports Exerc 25(5): 620-627.

19. Voltarelli FA, Gobatto CA, Mello MAR (2002).

Determination of anaerobic threshold in rats using the lactate minimum test. Braz J Med Biol Res 35: 1389-1394.
20. Voltarelli FA, Nunes WMS, Santiago V, Pauli JR, Garcia DR, Romero C, Silva AS, Mello MAR (2003) Determinação do Limiar Anaeróbio em Ratas Obesas com Glutamato Monossódico (MSG). Rev Logos (11): 84-93

21. Wasserman K, Mc Ilroy MB (1964) Detecting the threshold of anaerobic metabolism in cardiac patients during exercise. Am J Cardiol 14: 844-852. 